\title{
Invasive lobular carcinoma mammographic findings: correlation with age, breast composition, and tumour size
}

\author{
Corrado Tagliati ${ }^{1 A, B, C, D, E, F}$, Federico Cerimele ${ }^{2 B, D, E, F}$, Antonietta Di Martino ${ }^{10}$, Fabrizio Capone ${ }^{1 D}$, \\ Marialuisa Di Matteo ${ }^{10, F}$, Nevia Caputo ${ }^{1 D}$, Gabriella Lucidi Pressanti ${ }^{10}$, Ying Mingliang ${ }^{30, F}$, Silvia Baldassarre ${ }^{2 D}$, \\ Andrea Giovagnoni ${ }^{2 D}$, Gian Marco Giuseppetti2 ${ }^{2 A, D, E}$ \\ ${ }^{1}$ U.0. Radiologia, ASL Teramo, Italy \\ 2Department of Radiological Sciences, Azienda Ospedaliero Universitaria Ospedali Riuniti, Università Politecnica delle Marche, Ancona, Italy \\ ${ }^{3}$ Department of Radiology, Jinhua Municipal Central Hospital, Jinhua, Zhejiang, China
}

\section{Abstract}

Purpose: The aim of this study was to evaluate mammographic findings associated with invasive lobular carcinoma in different age groups, taking into account breast composition and tumour size.

Material and methods: A total of 1023 invasive lobular carcinoma preoperative mammograms were evaluated. According to the American College of Radiology Breast Imaging Reporting and Data System, cancer mammographic findings were classified as mass, calcifications, architectural distortion, and asymmetry, and breasts were assessed as dense ( $\mathrm{C}$ or $\mathrm{D}$ breast composition) or non-dense (A or B). The patient cohort was subdivided into 3 age groups $(<50,50-69, \geq 70$ years of age). In order to make the size and age groups dichotomous variables and to perform multiple regression analysis, a cut-off of $10 \mathrm{~mm}$ was chosen for tumour size, and $<50$-years-old and 50-69-years-old age groups were grouped together (<70-years-old age group).

Results: Significant results of multivariate analysis were the association between mass finding and non-dense breasts and size $\geq 10 \mathrm{~mm}(p<0.0001)$, between calcifications, and dense breasts, size $<10 \mathrm{~mm}$ and $<70$-years-old age group $(p<0.0001)$, between distortion and $<70$-years-old age group $(p=0.0366)$, and between asymmetry and $\geq 70$-years-old age group $(p=0.0090)$.

Conclusions: Various mammographic findings are differently associated with age group, breast composition, and tumour size.

Key words: invasive lobular carcinoma, breast composition, mammographic findings, age groups, tumour size.

\section{Introduction}

Breast cancer is the most common cancer in women, representing $30 \%$ of malignant tumours $[1,2]$. Invasive lobular carcinoma (ILC) accounts for $10-15 \%$ of all breast cancers and is the second most common histologic type after invasive ductal carcinoma [3]. Moreover, it seems that ILC incidence is increasing, particularly in young women, and this could be at least partially ascribed to the more prevalent use of E-cadherin staining in the diagnosis of ILC [4-6].

Mammography is still considered the first-line imaging modality of choice in women aged 40 years or over, and it should be performed in patients under 40 years old with clinically suspicious findings and/or ultrasonically suspicious findings [7-9]. Even in women with clinically significant focal and noncyclical breast pain or with pathologic

Correspondence address:

Corrado Tagliati, U.0. Radiologia, ASL Teramo, Dipartimento dei Servizi, Viale del Risorgimento, 1158, 64032 Atri, Teramo, Italy, phone: +39 0858707567 , fax: +39085 8707308, e-mail: corrado.tagliati@gmail.com

Authors' contribution:

A Study design · B Data collection · C Statistical analysis · D Data interpretation - E Manuscript preparation · F Literature search · G Funds collection 
nipple discharge aged greater than or equal to 30 years, mammography is usually considered appropriate as an initial imaging modality $[10,11]$.

Various articles evaluated invasive lobular carcinoma mammographic findings [12-19]. However, to our knowledge, no previous published studies assessed these findings taking into account patients' age, breast composition, and tumour size. Therefore, the aim of the present study is to evaluate the correlation between invasive lobular carcinoma mammographic findings and patients' age, breast composition, and tumour size.

\section{Material and methods}

From January 2013 to December 2019, about 75,000 mammograms were performed in our institution, and 4378 invasive breast cancers were resected. Inclusion criteria consisted of surgical intervention and postoperative pathologic assessment performed in our institution.

Exclusion criteria consisted of the following: male breast cancer $(n=36)$, personal history of breast cancer $(n=327)$, unavailability of mammography $(n=210)$ on our PACS (Picture Archiving and Communication System - Centricity GE PACS 4.0, General Electrics, USA), mammographically diagnosed cancer different from invasive lobular carcinoma $(n=2949)$, and negative mammography $(n=183)$. Therefore, 673 patients were included in the study. They were divided into 3 age groups: the first consisted of patients under 50 years of age, the second between 50 and 69 years old, and the third aged 70 years or over.

Within the study cohort, 399 (59.3\%) women were asymptomatic, while 278 (40.7\%) showed breast symptoms such as palpable breast mass, focal and noncyclical breast pain, pathologic nipple discharge, focal skin thickening, and/or retraction.

The study was approved by the institutional review board and the ethics committee of our institution (2020-55). Informed consent was obtained from each participant included in the study.

\section{Mammographic examination}

All mammograms were performed using the same machine (Selenia Value + Hologic, Marlborough, Massachusetts, USA). Mammographic examinations included craniocaudal (CC) and mediolateral oblique (MLO) views of each breast.

\section{Image assessment}

Two radiologists with at least 10 years of breast imaging experience, according to the American College of Radiology Breast Imaging Reporting and Data System (ACR BI-RADS ${ }^{\circledR}$ Atlas 2013), retrospectively assessed breast composition and mammographic findings in separate reading sessions, blinded to patient personal data, radiologic reports, and each other's assessments [20]. Disagreements were settled by consensus. When the 2 investigators did not reach consensus, mammographic images were evaluated by another radiologist with at least 30 years of experience, with the role of supervisor.

For blinding purposes, the mammograms were mixed into a stack of approximately 2000 other mammographies containing other types of malignancies, benign findings, and negative cases.

Image reading was carried out on standard views (CC, MLO).

According to the ACR BI-RADS ${ }^{\circledR}$ Atlas 2013, breast composition (BC) was categorized as " $\mathrm{A}$ " if the breast was almost entirely fatty, "B" if there were scattered areas of fibroglandular density, "C" if the breast was heterogeneously dense, or " $\mathrm{D}$ " if the breast was extremely dense.

Breast cancer mammographic findings were classified as follows: mass, calcification architectural distortion, and asymmetry [20].

\section{Histopathological findings}

Breast-conserving surgery or mastectomy specimens were used as the reference standard in order to classify breast cancers as invasive lobular carcinomas and to evaluate lesion size. Histological findings were classified according to the 2019 WHO classification [21].

\section{Statistical analysis}

Ordinal qualitative variables were analysed using the nonparametric Mann-Whitney $U$ test and the Kruskal-Wallis $H$-test. Quantitative variables were analysed using the parametric $t$-test and ANOVA. Only the main mammographic findings were analysed, whereas associated features were not. Breasts were evaluated as dense (C or D BC) or non-dense (A or B BC). In order to make the size and age group dichotomous and to perform multiple regression analysis, a cutoff of $10 \mathrm{~mm}$ was chosen for tumour size, and < 50-yearsold and 50-69-years-old age groups were grouped together ( $<70$-years-old age group). The variables found to be significant in univariate analysis using the non-parametric MannWhitney $U$ test were subsequently evaluated using multivariate analysis. Multiple regression with forward stepwise covariate selection was performed (with $\mathrm{p}$ values for entry and removal of 0.05 and 0.1 , respectively). The statistical significance level was set at $p<0.05$. All statistical analyses were performed using MedCalc Software v. 15.8 (Ostend, BEL).

\section{Results}

The mean age of the women in our study was $60.5 \pm 14.5$ years, range 31-89. The mean tumour size was $15.4 \pm 7.6 \mathrm{~mm}$.

Table 1 shows the tumour mean size in the 3 age groups, and Table 2 shows the mammographic findings related to BC in each age group. 
Mammographic sensitivity and specificity were, respectively, $85.6 \%$ and $82.4 \%$.

Tumour size showed to be significantly smaller in 50-69 age group $(p<0.001)$, and significantly larger in the $\geq 70$-years-old age group $(p<0.001)$.

Within the $<50$-years-old age group, the mass showed a statistically significant association with non-dense breast ( $p=0.0001)$, and calcifications were shown to be significantly associated with dense breast $(p=0.0004)$. Within the 50-69-years-old age group, mass showed a statistically significant association with non-dense breast $(p=0.0003)$, and calcifications were shown to be significantly associated with dense breast $(p<0.0001)$. Within the $\geq 70$-years-old age group, calcifications showed a statistically significant association with dense breast $(p<0.0001)$.

Considering only the 3 age subgroups of patients with non-dense breasts, distortion was shown to be significantly associated with 50-69-years-old age group ( $p=0.0427$ ). Considering only the 3 dense breast age subgroups, distortion was shown to be significantly associated with $<50$-years-old age group ( $p=0.0158$ ), and asymmetry was shown to be significantly associated with 50-69-years-old age group $(p=0.0001)$.

After making the size and age group dichotomous and using Mann-Whitney $U$ test, mass finding showed a statistically significant association with $\geq 70$-years-old age group $(p=0.0009)$, and a statistically significant inverse correlation with $<50$-years-old age group $(p=0.0257)$. Calcifications showed a statistically significant association with $<50$-yearsold age group $(p<0.0001)$ and a statistically significant inverse correlation with $\geq 70$-years-old age group $(p<0.0001)$. Distortion showed a statistically significant inverse correlation with $\geq 70$-years-old age group $(p=0.0373)$. Asymmetry showed a statistically significant association with $\geq 70$-yearsold age group $(p<0.0097)$ and a statistically significant inverse correlation with $<50$-years-old age group $(p<0.0001)$.

Mass showed a statistically significant association with non-dense breast $(p<0.0001)$, calcifications were significantly associated with dense breast $(p<0.0001)$, and asymmetry showed a statistically significant association with non-dense breast $(p<0.0287)$.

Mass showed a statistically significant association with tumour size $10 \mathrm{~mm}$ or larger $(p<0.0001)$ and calcifications were significantly associated with tumour size less than $10 \mathrm{~mm}(p<0.0001)$.

Significant results of multivariate analysis were the association between mass finding and non-dense breast and tumour size of $10 \mathrm{~mm}$ or larger $(p<0.0001)$, between calcifications, and dense breast, tumour size of less than $10 \mathrm{~mm}$ and $<70$-years-old age group ( $p<0.0001)$, and between asymmetry and $\geq 70$-years-old age group $(p=0.0090)$ (Figures 1-3).

\section{Discussion}

It is known that the sensitivity of mammography for breast cancer is mainly influenced by breast density, which has an inversely proportional correlation with age [22].

Table 1. Study population age groups and tumour size

\begin{tabular}{|l|c|}
\hline Age groups & Mean tumour size $(\mathrm{mm}) \pm \mathrm{DS}$ \\
\hline All patients, $n=673(100 \%)$ & $15.4 \pm 7.6$ \\
\hline$<50 \mathrm{yy}, n=213(31.6 \%)$ & $15.2 \pm 6.6$ \\
\hline $50-69 \mathrm{yy}, n=234(34.8 \%)$ & $12.3 \pm 6.2$ \\
\hline$\geq 70 \mathrm{yy}, n=226(33.6 \%)$ & $18.8 \pm 8.2$ \\
\hline
\end{tabular}

Table 2. Mammographic findings and breast composition in each age group

\begin{tabular}{|l|c|c|c|c|}
\hline Mammographic findings & \multicolumn{4}{|c|}{ Breast composition } \\
\hline$<50$ yy, $n=213(31.6 \%)$ & Non-dense breast, $n=56(26.3 \%)$ & \multicolumn{2}{c|}{ Dense breast, $n=157(73.7 \%)$} \\
\hline Mass, $n=114(53.5 \%)$ & 43 & $76.8 \%$ & 71 & $45.2 \%$ \\
\hline Calcifications, $n=43(20.2 \%)$ & 2 & $3.6 \%$ & 41 & $26.1 \%$ \\
\hline Architectural distortion, $n=53(24.9 \%)$ & 9 & $16.1 \%$ & 44 & $28.0 \%$ \\
\hline Asymmetry, $n=3(1.4 \%)$ & 2 & $3.6 \%$ & 1 & $0.7 \%$ \\
\hline $50-69$ yy, $n=234(34.8 \%)$ & Non-dense breast, $n=149(63.7 \%)$ & Dense breast, $n=85(36.3 \%)$ \\
\hline Mass, $n=133(56.9 \%)$ & 98 & $65.8 \%$ & 35 & $41.2 \%$ \\
\hline Calcifications, $n=29(12.4 \%)$ & 3 & $2.0 \%$ & 26 & $30.6 \%$ \\
\hline Architectural distortion, $n=49(20.9 \%)$ & 36 & $24.2 \%$ & 13 & $15.3 \%$ \\
\hline Asymmetry, $n=23(9.8 \%)$ & 12 & $8.0 \%$ & 11 & $12.9 \%$ \\
\hline$\geq 70$ yy, $n=226(33.6 \%)$ & Non-dense breast, $n=190(84.1 \%)$ & Dense breast, $n=36(15.9 \%)$ \\
\hline Mass, $n=155(68.6 \%)$ & 134 & $70.5 \%$ & 21 & $58.3 \%$ \\
\hline Calcifications, $n=9(4.0 \%)$ & 2 & $1.1 \%$ & 7 & $19.4 \%$ \\
\hline Architectural distortion, $n=36(15.9 \%)$ & 30 & $15.8 \%$ & 6 & $16.7 \%$ \\
\hline Asymmetry, $n=26(11.5 \%)$ & 24 & $12.6 \%$ & 2 & $5.6 \%$ \\
\hline
\end{tabular}



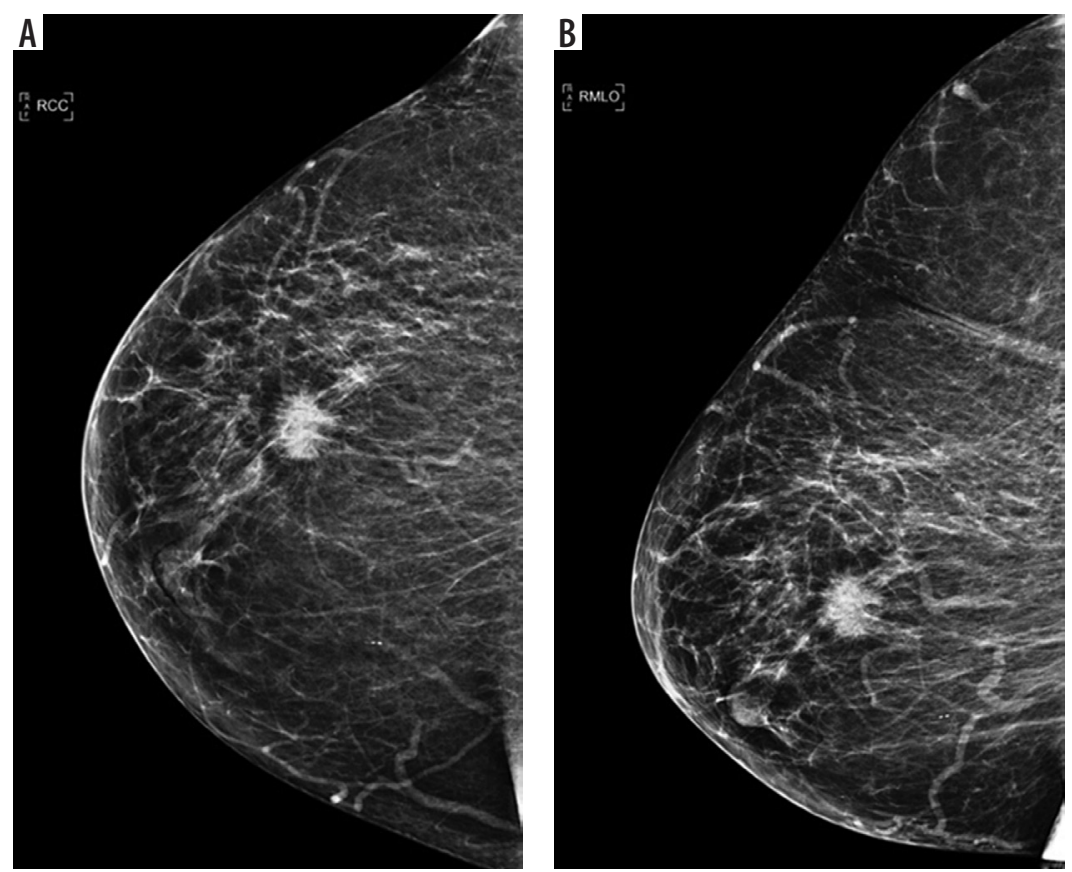

Figure 1.79-year-old woman with a $1.7 \mathrm{~cm}$ irregular spiculated mass in upper-outer quadrant of right breast: craniocaudal (A) and mediolateral oblique (B) views
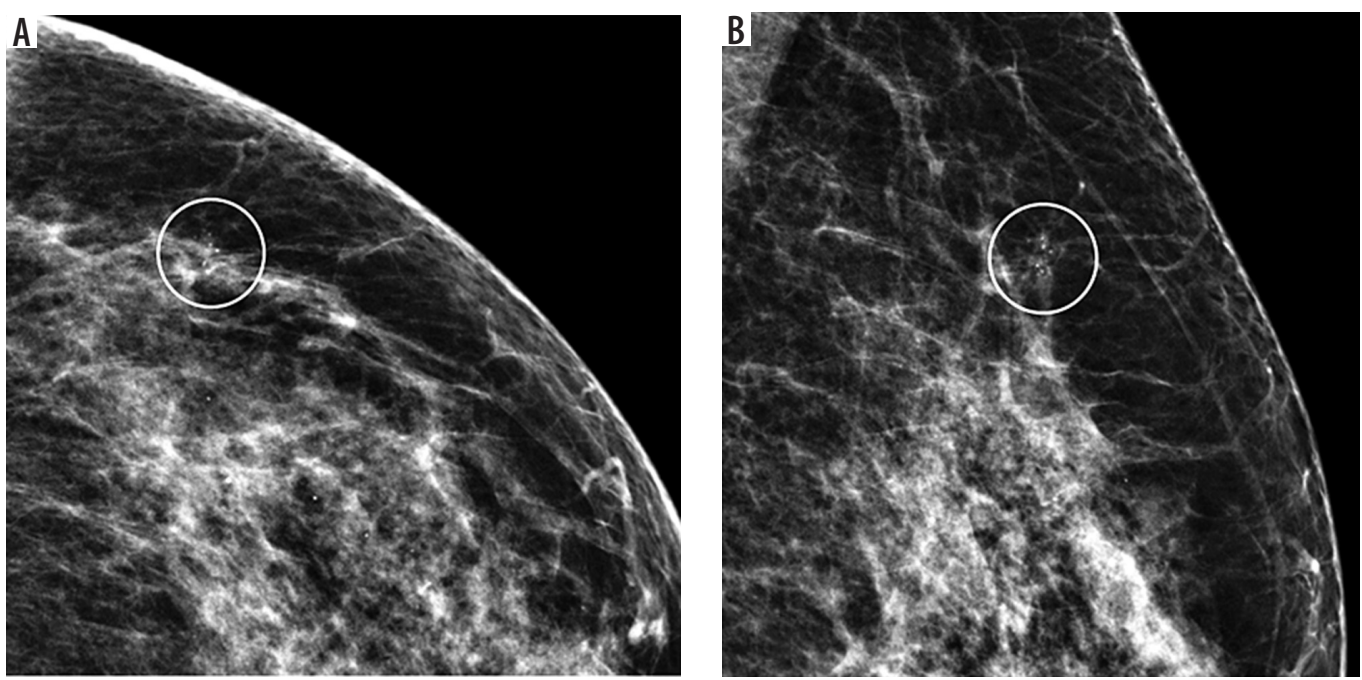

Figure 2. 43-year-old woman with grouped fine pleomorphic calcifications with an extension of $0.7 \mathrm{~cm}$ in upper-outer quadrant of left breast: craniocaudal (A) and mediolateral oblique (B) views

Moreover, mammographic diagnosis of ILC is reported to be more difficult than IDC, and this relatively low sensitivity has generated interest in other imaging modalities, such as magnetic resonance imaging, high-frequency ultrasonography, shear-wave elastography, tomosynthesis, and, more recently, contrast-enhanced digital mammography, in order to try to improve early and more accurate detection [6,16,23-33]. However, MLO and CC mammographic views are still considered the first-line imaging modalities of choice in women aged 40 years or over, both in a screening context and in the diagnostic assessment of patients with breast symptoms [7-9].

In the present study, in which the correlation between ILC mammographic findings and patients' age, breast composition and tumour size was evaluated, mass was found to be significantly associated with non-dense breast and size $10 \mathrm{~mm}$ or larger, and calcifications were significantly associated with dense breast, size less than $10 \mathrm{~mm}$, and $<70$ years of age. Moreover, distortion showed a statistically significant correlation with patients $<70$ years of age, and asymmetry with patients $\geq 70$ years of age.

Mammographic findings of ILC can be related to its characteristic growth pattern, typically described as rows of malignant cells that infiltrate breast tissue, but it may also be influenced by the relative paucity of connective tissue reaction and the frequent presence of multiple tumour foci scattered within normal breast parenchyma [34,35].

Within the cohort of patients, women aged $<50$ and $\geq 70$ years showed statistically significantly larger tumours, and these results could be at least partially as- 

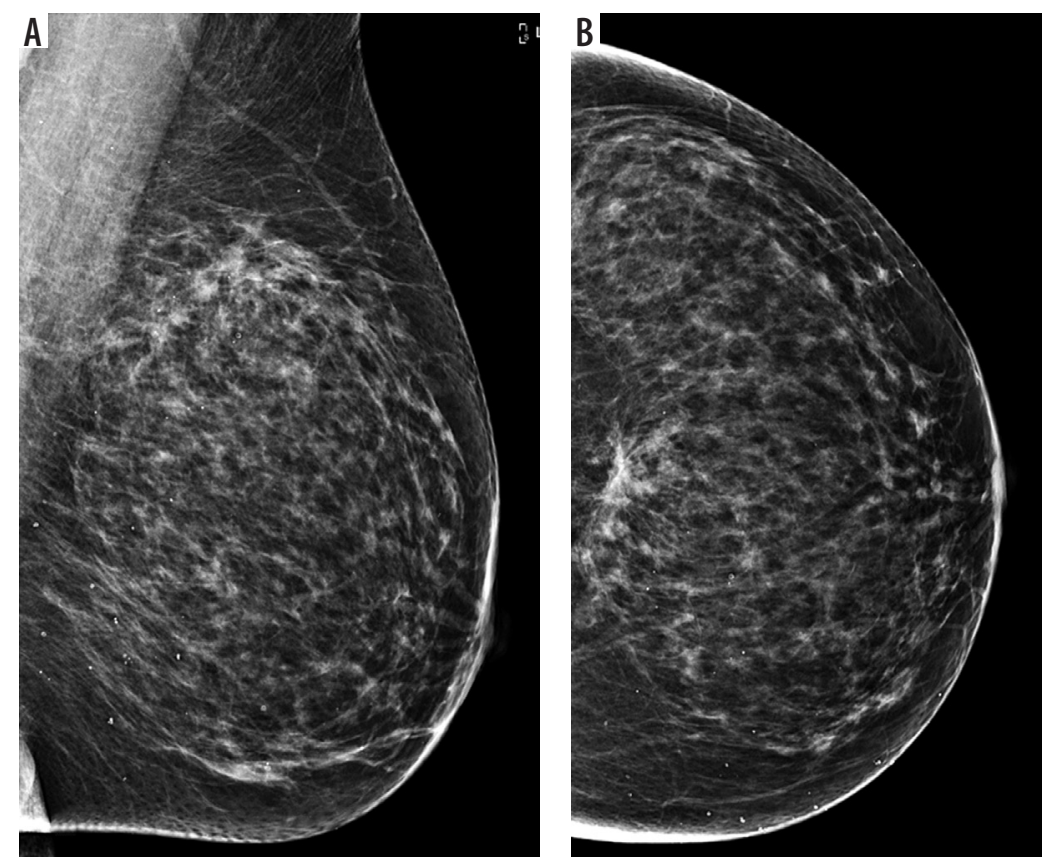

Figure 3. 47-year-old woman with a $3.2 \mathrm{~cm}$ architectural distortion in upper-inner quadrant of left breast: mediolateral oblique (A) and craniocaudal (B) views

cribed to the absence of routine examinations for most of these patients. In fact, previous studies reported that patients who perform routine breast examination show a smaller tumour size at diagnosis [36,37].

Based on previous studies, mass is the most frequent mammographic finding of ILC [12,14-17,38,39], and the present study confirmed this result. Moreover, as reported by various articles, the present study showed that architectural distortion, as well as asymmetry and calcifications, are not so rarely found $[12,14,16,17,40]$.

To our knowledge, only one previous study reported data about age distribution of mammographic findings and breast composition in a cohort of patients with ILC [19]. However, in this study, with about 107 ILCs, the patient population was subdivided into decades, tumour size was not reported, statistical analysis was not performed, and, taking into account the year of publication, the BI-RADS ${ }^{\circledR}$ breast imaging lexicon was not used; in fact, as is well known, the first edition of this reporting for mammography examination was released in 1993 [19,41,42].

The main limitation of the present study is the retrospective design. Moreover, the relatively limited number of

\section{References}

1. Özel D, Özel BD. Evaluating the role of strain ratio elastography in determining malignancy potential and calculating objective BIRADS US scores using ultrasonography and elastography features. Pol J Radiol 2018; 83: e268-e274.

2. Bayoumi D, Zaky M, Ibrahim DA, et al. The additive role of $1 \mathrm{H}$-magnetic resonance spectroscopic imaging to ensure pathological complete response after neoadjuvant chemotherapy in breast cancer patients. Pol J Radiol 2019; 84: e570-e580. patients did not allow a more detailed analysis of the mammographic findings. In fact, it was not possible to evaluate the morphology and the distribution of the calcifications nor the characteristics of the masses. In order to perform a morein-depth examination of these findings, a larger multicentric study is needed. However, to our knowledge, this is the first study in which a statistical analysis was performed to assess mammographic findings of patients with ILC, taking into account the age distribution of $\mathrm{BC}$ and tumour size.

\section{Conclusions}

This study emphasizes the heterogeneity of invasive lobular carcinoma mammographic findings, particularly showing that both mass and calcifications are significantly related to breast composition and tumour size, and that architectural distortion and asymmetry are significantly associated with patients' age.

\section{Conflict of interest}

The authors report no conflict of interest.

3. Luveta J, Parks RM, Heery DM, et al. Invasive lobular breast cancer as a distinct disease: implications for therapeutic strategy. Oncol Ther 2020; 8: 1-11.

4. Findlay-Shirras LJ, Lima I, Smith G, et al. Population trends in lobular carcinoma of the breast: the Ontario experience. Ann Surg Oncol 2020; 27: 4711-4719.

5. Dabbs DJ, Schnitt SJ, Geyer FC, et al. Lobular neoplasia of the breast revisited with emphasis on the role of E-cadherin immunohistochemistry. Am J Surg Pathol 2013; 37: e1-e11. 
6. Johnson K, Sarma D, Hwang ES. Lobular breast cancer series: imaging. Breast Cancer Res 2015; 17: 94.

7. Expert Panel on Breast Imaging; Mainiero MB, Moy L, Baron P, et al. ACR appropriateness criteria ${ }^{\circledR}$ breast cancer screening. J Am Coll Radiol 2017; 14: S383-S390.

8. The Royal College of Radiologists. Guidance on screening and symptomatic breast imaging, fourth edition. The Royal College of Radiologists, London 2019.

9. Expert Panel on Breast Imaging; Moy L, Heller SL, Bauley L, et al. ACR appropriateness criteria ${ }^{\circledR}$ palpable breast masses. J Am Coll Radiol 2017; 14: S203-S224.

10. Expert Panel on Breast Imaging; Holbrook AI, Moy L, Akin EA, et al. ACR appropriateness criteria ${ }^{\circledR}$ breast pain. J Am Coll Radiol 2018; 15: S276-S282.

11. Expert Panel on Breast Imaging; Lee SJ, Trikha S, Moy L, et al. ACR appropriateness criteria ${ }^{\circledR}$ evaluation of nipple discharge. J Am Coll Radiol 2017; 14: S138-S153.

12. Lopez JK, Bassett LW. Invasive lobular carcinoma of the breast: spectrum of mammographic, US, and MR imaging findings. Radiographics 2009; 29: 165-176.

13. Le Gal M, Ollivier L, Asselain B, et al. Mammographic features of 455 invasive lobular carcinomas. Radiology 1992; 185: 705-708.

14. Uchiyama N, Miyakawa K, Moriyama N, Kumazaki T. Radiographic features of invasive lobular carcinoma of the breast. Radiat Med 2001; 19: 19-25.

15. Cornford EJ, Wilson AR, Athanassiou E, et al. Mammographic features of invasive lobular and invasive ductal carcinoma of the breast: a comparative analysis. Br J Radiol 1995; 68: 450-453.

16. Hilleren DJ, Andersson IT, Lindholm K, Linnell FS. Invasive lobular carcinoma: mammographic findings in a 10-year experience. Radiology 1991; 178: 149-154.

17. Helvie MA, Paramagul C, Oberman HA, Adler DD. Invasive lobular carcinoma. Imaging features and clinical detection. Invest Radiol 1993; 28: 202-207.

18. Newstead GM, Baute PB, Toth HK. Invasive lobular and ductal carcinoma: mammographic findings and stage at diagnosis. Radiology 1992; 184: 623-627.

19. Adler OB, Engel A. Invasive lobular carcinoma. Mammographic pattern. Rofo 1990; 152: 460-462.

20. D’Orsi CJ, Sickles EA, Mendelson EB, et al. ACR BI-RADS ${ }^{\circledR}$ Atlas, Breast Imaging Reporting and Data System. $5^{\text {th }}$ ed. American College of Radiology, Reston 2013

21. Hoon Tan P, Ellis I, Allison K, et al. The 2019 WHO classification of tumours of the breast. Histopathology 2020; 77: 181-185.

22. Yadav P, Chauhan S. Effectivity of combined diffusion-weighted imaging and contrast-enhanced MRI in malignant and benign breast lesions. Pol J Radiol 2018; 83: e82-e93.

23. Ha SM, Chae EY, Cha JH, et al. Breast MR imaging before surgery: outcomes in patients with invasive lobular carcinoma by using propensity score matching. Radiology 2018; 287: 771-777.

24. Ha SM, Chae EY, Cha JH, et al. Long-term survival outcomes in invasive lobular carcinoma patients with and without preoperative MR imaging: a matched cohort study. Eur Radiol 2019; 29: 2526-2534.

25. Derias M, Subramanian A, Allan S, et al. The role of magnetic resonance imaging in the investigation and management of invasive lobular carcinoma - a 3-year retrospective study in two district general hospitals. Breast J 2016; 22: 384-389.

26. Wen X, Yu Y, Yu X, et al. Correlations between ultrasonographic findings of invasive lobular carcinoma of the breast and intrinsic subtypes. Ultraschall Med 2019; 40: 764-770.

27. Sim YT, Vinnicombe S, Whelehan P, et al. Value of shear-wave elastography in the diagnosis of symptomatic invasive lobular breast cancer. Clin Radiol 2015; 70: 604-609.

28. Brkljačić B, Divjak E, Tomasović-Lončarić Č, et al. Shear-wave sonoelastographic features of invasive lobular breast cancers. Croat Med J 2016; 57: 42-50.

29. Chamming's F, Kao E, Aldis A, et al. Imaging features and conspicuity of invasive lobular carcinomas on digital breast tomosynthesis. $\mathrm{Br}$ J Radiol 2017; 90: 20170128.

30. Grubstein A, Rapson Y, Morgenstern S, et al. Invasive lobular carcinoma of the breast: appearance on digital breast tomosynthesis. Breast Care (Basel) 2016; 11: 359-362.

31. Mariscotti G, Durando M, Houssami N, et al. Digital breast tomosynthesis as an adjunct to digital mammography for detecting and characterising invasive lobular cancers: a multi-reader study. Clin Radiol 2016; 71: 889-895.

32. Patel BK, Davis J, Ferraro C, et al. Value added of preoperative contrast-enhanced digital mammography in patients with invasive lobular carcinoma of the breast. Clin Breast Cancer 2018; 18 : e1339-e1345.

33. Amato F, Bicchierai G, Cirone D, et al. Preoperative loco-regional staging of invasive lobular carcinoma with contrast-enhanced digital mammography (CEDM). Radiol Med 2019; 124: 1229-1237.

34. Qayyum A, Birdwell RL, Daniel BL, et al. MR imaging features of infiltrating lobular carcinoma of the breast: histopathologic correlation. AJR Am J Roentgenol 2002; 178: 1227-1232.

35. Levrini G, Mori CA, Vacondio R, et al. MRI patterns of invasive lobular cancer: T1 and T2 features. Radiol Med 2008; 113: 1110-1125.

36. Schattner E. Correcting a decade of negative news about mammography. Clin Imaging 2020; 60: 265-270.

37. James TA, Wade JE, Sprague BL. The impact of mammographic screening on the surgical management of breast cancer. J Surg Oncol 2016; 113: 496-500.

38. Bazzocchi M, Facecchia I, Zuiani C, et al. Diagnostic imaging of lobular carcinoma of the breast: mammographic, ultrasonographic and MR findings. Radiol Med 2000; 100: 436-443.

39. Veltman J, Boetes C, van Die L, et al. Mammographic detection and staging of invasive lobular carcinoma. Clin Imaging 2006; 30: 94-98.

40. Albayrak ZK, Onay HK, Karatağ GY, Karatağ O. Invasive lobular carcinoma of the breast: mammographic and sonographic evaluation. Diagn Interv Radiol 2011; 17: 232-238.

41. Gemici AA, Inci E. Agreement between dynamic contrast-enhanced magnetic resonance imaging and pathologic tumour size of breast cancer and analysis of the correlation with BI-RADS descriptors. Pol J Radiol 2019; 84: e616-e624.

42. Koziełek K, Stranz-Walczak N, Gajdzis P, Karmelita-Katulska K. Evaluation of the positive predictive value (PPV3) of ACR BI-RADS category 4 and 5 based on the outcomes of invasive diagnostic office in an outpatient clinic. Pol J Radiol 2019; 84: e185-e189. 